\title{
Crystal structure of the superconducting phase of sulfur hydride
}

\author{
Mari Einaga ${ }^{1 \star \dagger}$, Masafumi Sakata', Takahiro Ishikawa ${ }^{1}$, Katsuya Shimizu $^{1 \dagger}$, Mikhail I. Eremets ${ }^{2 \dagger}$, \\ Alexander P. Drozdov' ${ }^{2}$ Ivan A. Troyan ${ }^{2}$, Naohisa Hirao ${ }^{3}$ and Yasuo Ohishi ${ }^{3}$
}

\begin{abstract}
A superconducting critical temperature above $200 \mathrm{~K}$ has recently been discovered in $\mathrm{H}_{2} \mathrm{~S}$ (or $D_{2} S$ ) under high hydrostatic pressure $^{1,2}$. These measurements were interpreted in terms of a decomposition of these materials into elemental sulfur and a hydrogen-rich hydride that is responsible for the superconductivity, although direct experimental evidence for this mechanism has so far been lacking. Here we report the crystal structure of the superconducting phase of hydrogen sulfide (and deuterium sulfide) in the normal and superconducting states obtained by means of synchrotron X-ray diffraction measurements, combined with electrical resistance measurements at both room and low temperatures. We find that the superconducting phase is mostly in good agreement with the theoretically predicted body-centred cubic (bcc) structure for $\mathrm{H}_{3} \mathrm{~S}^{3}$. The presence of elemental sulfur is also manifest in the X-ray diffraction patterns, thus proving the decomposition mechanism of $\mathrm{H}_{2} \mathrm{~S}$ to $\mathrm{H}_{3} \mathrm{~S}+\mathrm{S}$ under pressure ${ }^{4-6}$.
\end{abstract}

Recently, a very high $T_{c}$ of $200 \mathrm{~K}$ has been discovered in the hydrogen sulfide system ${ }^{1,2}$. This work was initiated by the prediction of a substantial superconductivity in $\mathrm{H}_{2} \mathrm{~S}$ (ref. 7), which in turn arises from the idea that hydrogen-dominant metallic alloys might be superconductors with high critical temperature, similar to pure metallic hydrogen ${ }^{8}$.

The superconducting transition was proved by the sharp drop of the resistance to zero, a strong isotope effect in a study of $\mathrm{D}_{2} \mathrm{~S}$, a shift of the superconducting transition with magnetic field, and finally by measuring the magnetic susceptibility and magnetization. As a likely explanation, the authors ${ }^{1,2}$ suggested that $\mathrm{H}_{2} \mathrm{~S}$ decomposes under pressure (with the assistance of temperature) to pure sulfur and some sulfur hydride with a higher content of hydrogen (such as $\mathrm{SH}_{4}$ or similar). At the same time, a theoretical work appeared which considered a different starting material $\left(\mathrm{H}_{2} \mathrm{~S}\right)_{2} \mathrm{H}_{2}$ (stoichiometry $\mathrm{H}_{3} \mathrm{~S}$ ) and found $\mathrm{R} 3 \mathrm{~m}$ and $\mathrm{Im}-3 \mathrm{~m}$ structures under pressure above $111 \mathrm{GPa}$ and $180 \mathrm{GPa}$, respectively ${ }^{3}$. These structures and other stoichiometric compounds were further carefully studied theoretically by different groups in numerous works ${ }^{4,6,9-25}$ and $T_{\mathrm{c}} \sim 200 \mathrm{~K}$ was consistently obtained for the $\mathrm{Im}-3 \mathrm{~m}$ structure. The calculated $T_{c}$, as well as its pressure dependence ${ }^{9}$, are close to the experimental data ${ }^{1,2}$. This suggests that the high $T_{c}$ observed in the experiments relates not to $\mathrm{H}_{2} \mathrm{~S}$, but to the $\mathrm{H}_{3} \mathrm{~S}$ in the $\mathrm{Im}-3 m$ structure. Later calculations supported this idea: $\mathrm{H}_{2} \mathrm{~S}$ is indeed unstable at high pressures and should decompose to sulfur and higher hydrides, most likely to $\mathrm{H}_{3} \mathrm{~S}^{4,6,12}$. The goal of the present work is to check experimentally the structure of the superconducting hydrogen sulfide and compare it with the theoretically predicted structure.

Samples were prepared in the same way as described in refs 1 , $2-\mathrm{H}_{2} \mathrm{~S}$ was loaded at temperatures of $\sim 200 \mathrm{~K}$, then the pressure was increased to $\sim 150-170 \mathrm{GPa}$ and the sample was annealed at room temperature. Typical X-ray diffraction (XRD) images of sulfur hydride and sulfur deuteride pressurized to $150-173 \mathrm{GPa}$ are shown in Fig. 1. The XRD patterns of sulfur hydride and sulfur deuteride samples do not differ from each other. The diffraction patterns seem to be produced by two major phases. This clearly follows from the different pressure dependence of the peaks (Fig. 2 and Supplementary Fig. 3) and different variation of intensities while scanning the sample over its diameter (Supplementary Fig. 1): one group is fitted by elemental sulfur of the $\beta$-Po structure $^{26}$ and another group is described by the bcc structure of $\mathrm{H}_{3} \mathrm{~S}$ from the theoretical work ${ }^{3}$. We can conclude that $\mathrm{H}_{2} \mathrm{~S}$ $\left(\mathrm{D}_{2} \mathrm{~S}\right)$ solid most likely decomposes under pressure via the route: $3 \mathrm{H}_{2} \mathrm{~S} \rightarrow 2 \mathrm{H}_{3} \mathrm{~S}+\mathrm{S}$.

The pressure dependence of the atomic volume, $V_{\mathrm{atm}}$, of sulfur hydride and sulfur deuteride are shown in Fig. 2c. It is fitted by a first-order Birch equation of state ${ }^{27}$ with the bulk modulus $B_{0}=506(30) \mathrm{GPa}$, and its pressure derivative $B_{0}^{\prime}=6$ (fixed). The value of the experimentally observed $V_{\text {atm }}$ is slightly larger, but the compressibility is in good agreement with Duan's calculation ${ }^{3}$. The pressure dependence of the normalized atomic volume $V / V_{0}$ of elemental sulfur in the $\beta$-Po structure is shown in Supplementary Fig. 3. It is in a good agreement with the experimental data of ref. 26 at high pressures $P>170 \mathrm{GPa}$, and with our density functional theory calculations (see Methods).

Our powder XRD measurements do not allow us to distinguish between the predicted bcc structures: $I m-3 m$ and $R 3 m$. In these structures the positions of the sulfur atoms are the same and the only difference is the position of the hydrogen atoms: hydrogen atoms are situated symmetrically between neighbouring sulfur atoms in the $I m-3 m$ structure and slightly asymmetrically in the $R 3 m$ structure (Supplementary Fig. 2). However, the position of the hydrogen atoms cannot be determined from the powder measurements, as hydrogen atoms are extremely weak scatterers.

The low-temperature data help with further analysis. We measured simultaneously the XRD and electrical resistance in the same set-up ${ }^{28}$ (Fig. 3). The transition to the superconducting state was determined from the sharp drop of the resistance (Fig. 3a,b). We found that the normal and the superconducting state have the same structure, as the XRD patterns are the same

${ }^{1}$ KYOKUGEN, Graduate School of Engineering Science, Osaka University, Machikaneyamacho 1-3, Toyonaka, Osaka 560-8531, Japan. ${ }^{2}$ Max-Planck Institut für Chemie, Hahn-Meitner-Weg 1, 55128 Mainz, Germany. ${ }^{3}$ JASRI/SPring-8, 1-1-1, Sayo-cho, Sayo-gun, Hyogo 679-5198, Japan. ${ }^{\dagger}$ These authors

contributed equally to this work. *e-mail: einaga@hpr.stec.es.osaka-u.ac.jp 


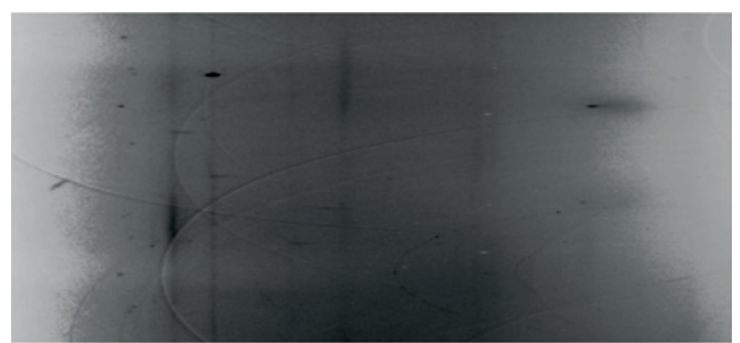

c

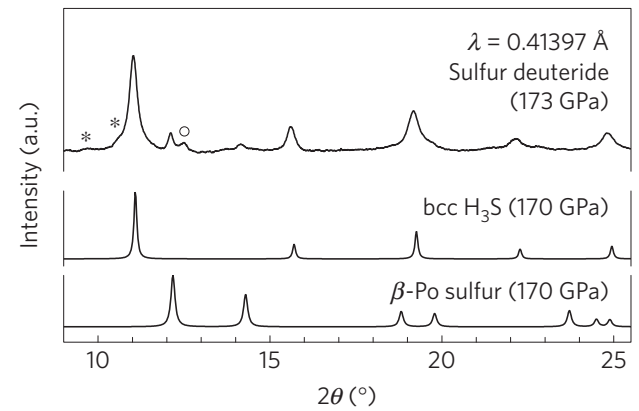

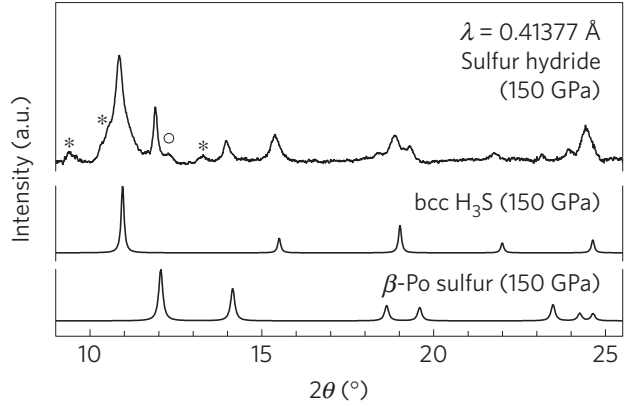

d

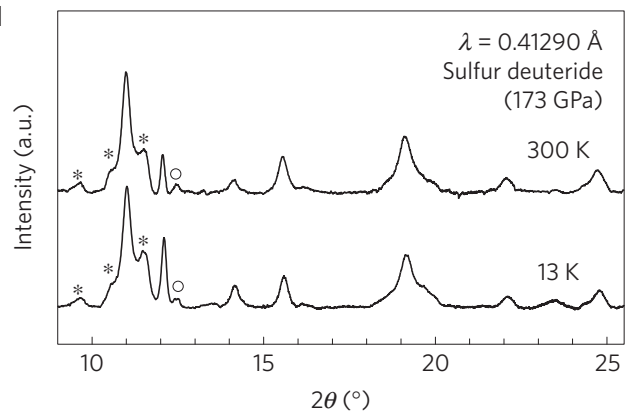

Figure 1 | XRD of the sulfur hydride and sulfur deuteride samples. a, Unrolled powder diffraction image of sulfur hydride at $150 \mathrm{GPa}$ at room temperature recorded on the imaging plate. b,c, Integrated XRD patterns obtained with subtraction of the background for sulfur hydride (b) and sulfur deuteride (c). The patterns of bcc $\mathrm{H}_{3} \mathrm{~S}$ and $\beta$-Po elemental sulfur at $150 \mathrm{GPa}$ and $170 \mathrm{GPa}$ calculated according to refs 5,26 are shown beneath the experimentally obtained patterns. Stars indicate peaks that do not belong to the sample, as follows from the scan over the sample (Supplementary Fig. 1): these peaks remain unchanged, whereas the sample peaks change with the radius of the sample both in position and intensity. Open circles indicate a reflection from the high-pressure phase IV of elemental sulfur (incommensurately modulated body-centred monoclinic structure). $\mathbf{d}$, XRD patterns of sulfur deuteride at $173 \mathrm{GPa}$ at $300 \mathrm{~K}$ and $13 \mathrm{~K}$. The peaks marked by stars are not reflections from the sample. The results of analyses are shown in Supplementary Table 1.
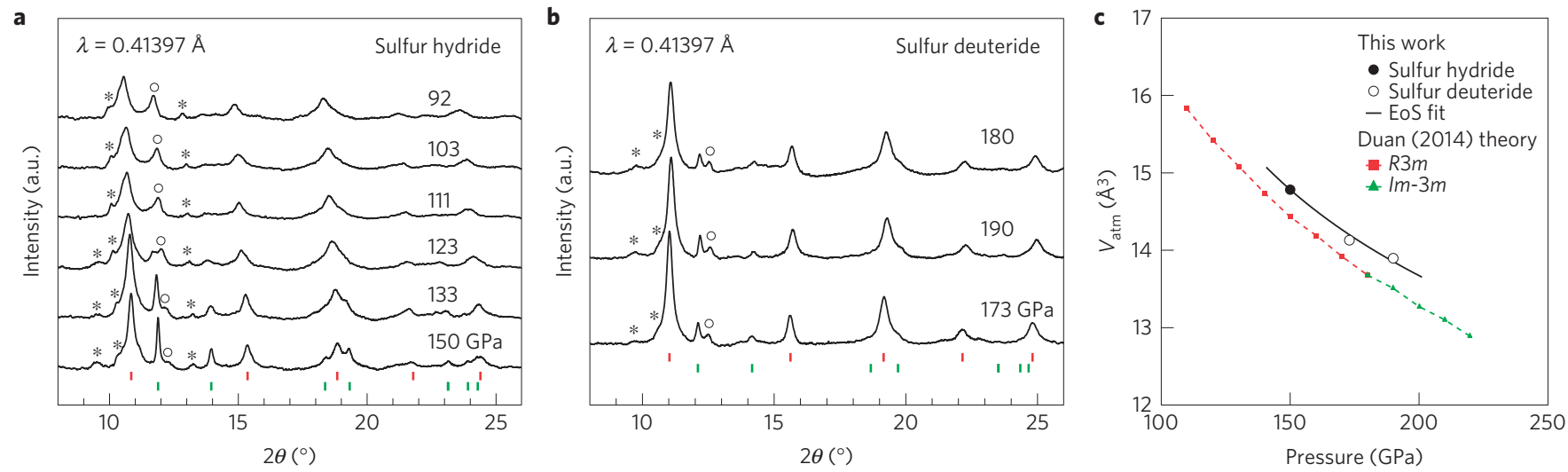

Figure 2 | Pressure dependence of XRD in sulfur hydride and sulfur deuteride samples. a,b, XRD patterns taken at room temperature and different pressures for sulfur hydride (a) and sulfur deuteride (b). Upper (red) and lower (green) ticks indicate the peak positions of the predicted bcc structure of

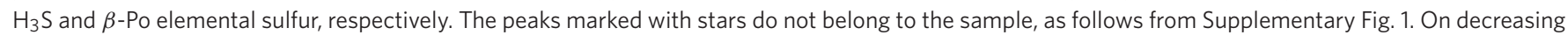
the pressure in sulfur hydride, the phase transition of elemental sulfur is clearly observed-the peak from $\beta$-Po sulfur gradually disappears and that from phase IV (open circle) is enhanced. c, Pressure dependence of the atomic volume of sulfur hydride and sulfur deuteride. The experimental data were obtained with increasing pressure and are fitted with a first-order Birch equation of state (black solid line). The volumes of hexagonal ( $\mathrm{R} 3 m$ ) and bcc $(\mathrm{Im}-3 \mathrm{~m})$ phases obtained from the theoretical work ${ }^{3}$ are shown as filled squares and filled triangles, respectively, connected with broken lines. The estimated standard deviations are smaller than the size of the symbols.

at room and low temperatures (Fig. 1d). Moreover, the structure of the sample does not change visibly over the pressure range $92-173 \mathrm{GPa}$. This is in a contrast to the dependence of the critical temperature on pressure, which has a pronounced kink at $150 \mathrm{GPa}$ for $\mathrm{H}_{3} \mathrm{~S}$ and $160 \mathrm{GPa}$ for $\mathrm{D}_{3} \mathrm{~S}$ (Fig. 3c). This kink finds a natural explanation in the theoretical predictions $s^{9,23}$ : the pressure dependence of the critical superconducting temperature is different in the $R 3 m$ phase at lower pressures and in the $I m-3 m$ phase at higher pressures. Our XRD measurements support this interpretation, as $R 3 m$ and $I m-3 m$ phases differ only in the ordering of the hydrogen atoms, and the same XRD patterns should be the same in the both pressure domains. Thus, one can conclude that the highest critical temperature of $203 \mathrm{~K}$ (ref. 2) corresponds to the Im-3m phase. 

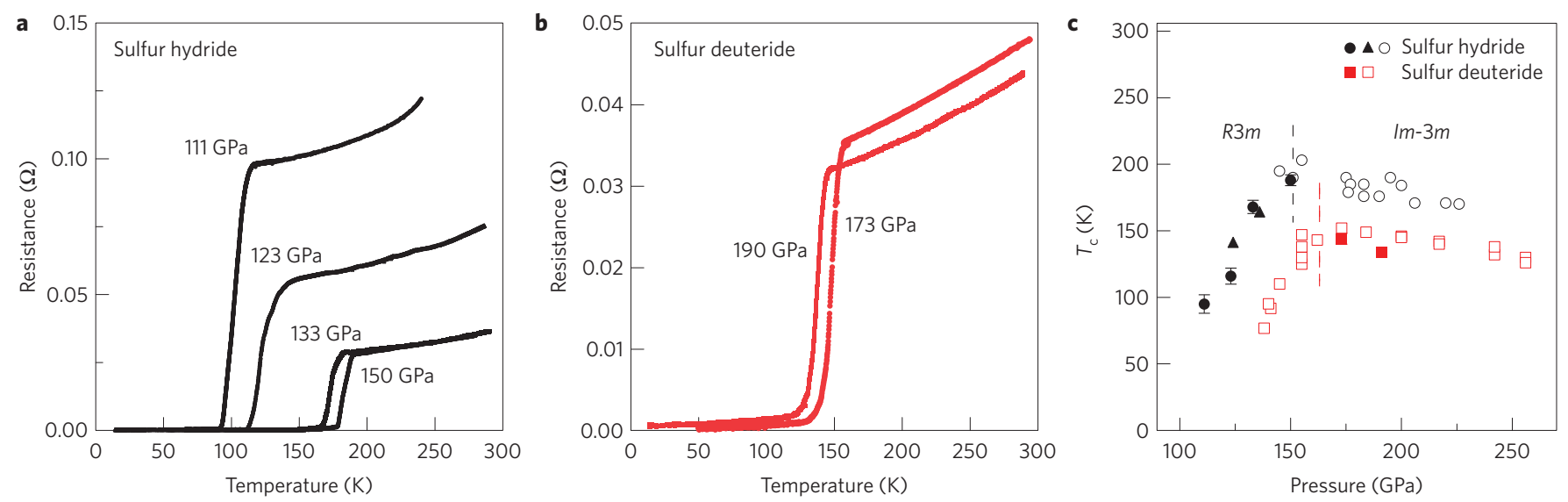

Figure $\mathbf{3}$ | Pressure dependence of the superconducting transition in sulfur hydride and sulfur deuteride. $\mathbf{a}, \mathbf{b}$, Temperature dependence of resistance in sulfur hydride (on decreasing pressure) (a) and sulfur deuteride (on increasing pressure) (b). c, Pressure dependence of critical temperature of superconductivity $T_{\mathrm{c}}$ of sulfur hydride (black points) and sulfur deuteride (red points). Open circles and squares are taken from ref. 2. The points marked with filled symbols are from the present work: the circles represent data on decreasing pressure, the squares and triangles on increasing pressure. Broken lines (black for sulfur hydride and red for sulfur deuteride) indicate the phase boundary between the $R 3 m$ and $I m$ - $3 m$ structural phases. The error bars indicate the difference between the onset temperature $T_{\mathrm{c}}$ and the zero-resistance temperature at each pressure in $\mathbf{a}$ and $\mathbf{b}$.

\section{Methods}

Methods, including statements of data availability and any associated accession codes and references, are available in the online version of this paper.

Received 9 September 2015; accepted 12 April 2016; published online 9 May 2016

\section{References}

1. Drozdov, A. P., Eremets, M. I. \& Troyan, I. A. Conventional superconductivity at $190 \mathrm{~K}$ at high pressures. Preprint at http://arxiv.org/abs/1412.0460 (2014).

2. Drozdov, A. P., Eremets, M. I., Troyan, I. A., Ksenofontov, V. \& Shylin, S. I. Conventional superconductivity at 203 kelvin at high pressures in the sulfur hydride system. Nature 525, 73-77 (2015).

3. Duan, D. et al. Pressure-induced metallization of dense $\left(\mathrm{H}_{2} \mathrm{~S}\right)_{2} \mathrm{H}_{2}$ with high- $\mathrm{T}_{c}$ superconductivity. Sci. Rep. 4, 6968 (2014).

4. Bernstein, N., Hellberg, C. S., Johannes, M. D., Mazin, I. I. \& Mehl, M. J. What superconducts in sulfur hydrides under pressure, and why. Phys. Rev. B 91, 060511(R) (2015)

5. Duan, D. et al. Pressure-induced decomposition of solid hydrogen sulfide. Phys. Rev. B 91, 180502(R) (2015).

6. Errea, I. et al. High-pressure hydrogen sulfide from first principles: a strongly anharmonic phonon-mediated superconductor. Phys. Rev. Lett. 114, 157004 (2015).

7. Li, Y., Hao, J., Liu, H., Li, Y. \& Ma, Y. The metallization and superconductivity of dense hydrogen sulfide. J. Chem. Phys. 140, 174712 (2014).

8. Ashcroft, N. W. Hydrogen dominant metallic alloys: high temperature superconductors? Phys. Rev. Lett. 92, 187002 (2004).

9. Akashi, R., Kawamura, M., Tsuneyuki, S., Nomura, Y. \& Arita, R. First-principles study of the pressure and crystal-structure dependences of the superconducting transition temperature in compressed sulfur hydrides. Phys. Rev. B 91, 224513 (2015).

10. Flores-Livas, J. A., Sanna, A. \& Gross, E. K. U. High temperature superconductivity in sulfur and selenium hydrides at high pressure. Eur. Phys. J. B 89, 63 (2016).

11. Ge, Y., Zhang, F. \& Yao, Y. Possible superconductivity approaching ice point. Preprint at http://arxiv.org/abs/1507.08525 (2015).

12. Papaconstantopoulos, D. A., Klein, B. M., Mehl, M. J. \& Pickett, W. E. Cubic $\mathrm{H}_{3} \mathrm{~S}$ around $200 \mathrm{GPa}$ an atomic hydrogen superconductor stabilized by sulfur. Phys. Rev. B 91, 184511 (2015).

13. Li, Y. et al. Dissociation products and structures of solid $\mathrm{H}_{2} \mathrm{~S}$ at strong compression. Phys. Rev. B 93, 020103(R) (2016).

14. Quan, Y. \& Pickett, W. E. Van Hove singularities and spectral smearing in high-temperature superconducting $\mathrm{H}_{3} \mathrm{~S}$. Phys. Rev. Lett. 93, 104526 (2016).
15. Bianconi, A. \& Jarlborg, T. Lifshitz transitions and zero point lattice fluctuations in sulfur hydride showing near room temperature superconductivity. Nov. Supercond. Mater. 1, 37-49 (2015).

16. Nicol, E. J. \& Carbotte, J. P. Comparison of pressurized sulfur hydride with conventional superconductors. Phys. Rev. B 91, 220507(R) (2015).

17. Degtyarenko, N. \& Mazur, E. Causes of high-temperature superconductivity in the hydrogen sulfide electron-phonon system. Preprint at http://arxiv.org/abs/1507.05749 (2015).

18. Durajski, A. P., Szczéşniak, R. \& Li, Y. Non-BCS thermodynamic properties of $\mathrm{H}_{2} \mathrm{~S}$ superconductor. Physica C 515, 1-6 (2015).

19. Durajski, A. P., Szczéşniak, R. \& Pietronero, L. High-temperature study of superconducting hydrogen and deuterium sulfide. Ann. Phys. http://dx.doi.org/10.1002/andp.201500316 (2015).

20. Gor'kov, L. \& Kresin, V. Pressure and high $T_{\mathrm{c}}$ superconductivity: applications to sulfur hydrides. Preprint at http://arxiv.org/abs/1511.06926 (2015).

21. Hirsch, J. E. \& Marsiglio, F. Hole superconductivity in $\mathrm{H}_{2} \mathrm{~S}$ and other sulfides under high pressure. Physica C 511, 45-49 (2015).

22. Băacký, P. On the mechanism of high-temperature superconductivity in hydrogen sulfide at $200 \mathrm{GPa}$ : transition into superconducting anti-adiabatic state in coupling to H-vibrations. Results Phys. 6, 1-2 (2016).

23. Errea, I. et al. Quantum hydrogen-bond symmetrization in the superconducting hydrogen sulfide system. Nature 532, 81-84 (2016).

24. Akashi, R., Sano, W., Arita, R. \& Tsuneyuki, S. Possible Magnéli phases and self-alloying in the superconducting sulfur hydride. Preprint at http://arxiv.org/abs/1512.06680 (2015).

25. Ishikawa, T. et al. Superconducting $\mathrm{H}_{5} \mathrm{~S}_{2}$ phase in sulfur-hydrogen system under high-pressure. Sci. Rep. 6, 23160 (2016).

26. Luo, H., Greene, R. G. \& Ruoff, A. L. $\beta$-Po phase of sulfur at 162 GPa: X-ray diffraction study to 212 GPa. Phys. Rev. Lett. 71, 2943-2946 (1993).

27. Birch, F. Finite strain isotherm and velocities for single-crystal and polycrystalline $\mathrm{NaCl}$ at high pressures and $300^{\circ} \mathrm{K}$. J. Geophys. Res. 83, 1257-1268 (1978).

28. Matsuoka, T. et al. Pressure-induced reentrant metallic phase in lithium. Phys. Rev. B 89, 144103 (2014).

\section{Acknowledgements}

This work was performed under proposal No. 2015A0112 of the SPring-8. This research was supported by Japan Society for the Promotion of Science Grant-in-Aid for Specially Promoted Research, No. 26000006, JSPS KAKENHI, Grant-in-Aid for Young Scientists (B), No.15K17707 and the European Research Council 2010-Advanced Grant 267777.

\section{Author contributions}

M.E. took part in all the XRD measurements, data interpretation and writing the manuscript. M.S. performed the cryogenic operations and XRD data collection. K.S. 
performed in situ electrical resistance measurements in the XRD measurements and writing the manuscript. T.I. performed support calculations for the data interpretation. M.I.E. designed the study and participated in the XRD experiments and writing of the manuscript. A.P.D. prepared the sample in a diamond anvil cell for all the experiments

I.A.T. participated in building the Raman set-up. N.H. and Y.O. performed the

optimization of synchrotron XRD and cryogenic operations. M.E., K.S. and M.I.E. contributed equally to this paper.

\section{Additional information}

Supplementary information is available in the online version of the paper. Reprints and permissions information is available online at www.nature.com/reprints.

Correspondence and requests for materials should be addressed to M.E.

\section{Competing financial interests}

The authors declare no competing financial interests. 


\section{Methods}

The sample and electrical probes were prepared by similar method to ref. 2 . Angle-dispersive powder XRD measurements were carried out at SPring-8 (beamline BL10XU) with a monochromatic beam of energy $\sim 30.0 \mathrm{keV}$ $(\lambda \sim 0.412-0.414 \AA)$. XRD and electrical resistance were measured simultaneously with the aid of a cryostat ${ }^{28}$. The diffraction patterns were recorded using an imaging plate with an exposure time between 120 and 300 s. Four-probe electrica measurements were performed with an a.c.-resistance bridge (Linear Research, LR-700). We determined the pressure-volume dependence of $\beta$-Po sulfur by means of first-principles calculations based on density functional theory. The Quantum ESPRESSO code $^{29}$ was used for the calculations, in which the

Perdew-Burke-Ernzerhof generalized gradient approximation ${ }^{30}$ and the Vanderbilt ultrasoft pseudopotential ${ }^{31}$ were employed. The $k$-space integration over the Brillouin zone was performed on a $24 \times 24 \times 24$ grid, and the energy cutoff of the plane wave basis was set at 80 Ry.
Data availability. Raw data were generated at the SPring-8 synchrotron radiation facility (beamline BL10XU). Derived data supporting the findings of this study are available from the corresponding author on request.

\section{References}

29. Giannozzi, P. et al. QUANTUM ESPRESSO: a modular and open-source software project for quantum simulations of materials. J. Phys. Condens. Matter 21, 395502 (2009)

30. Perdew, J. P., Burke, K. \& Ernzerhof, M. Generalized gradient approximation made simple. Phys. Rev. Lett. 77, 3865-3868 (1996).

31. Vanderbilt, D. Soft self-consistent pseudopotentials in a generalized eigenvalue formalism. Phys. Rev. B 41, 7892-7895 (1990). 